\section{Nature of the Milk-Ejection Process}

THE fact that extracts of the posterior lobe of the pituitary gland will cause expulsion of milk from the mammary glands of lactating animals has been known for some time ${ }^{1,2}$. The suggestion that the oxytocic factor is involved in normal milk let-down was made by Ely and Petersen ${ }^{3}$, though Turner had proposed a theory of milk ejection earlier ${ }^{4}$ and with Cooper ${ }^{5}$ presented evidence for the existence of a separate milk-ejection hormone. This evidence was based on the use of a lactating rabbit as an assay animal, and showed that the milk-ejecting activity of whole pituitrin is greater than that of purified oxytocin for a given oxytocic activity, and that the purified pressor preparation is more active than it should be for its oxytocic content.

Using the lactating sow as an assay animal and milking her by machine, I have confirmed the observations of Turner and Cooper on the milkejecting activity of commercial purified pressor preparation. Through the generosity of Dr. du Vigneaud, a sample of purified oxytocin assaying 500 oxytocic units per mgm. was made available for milk-ejection assay. This sample had been prepared by the method of Livermore and du Vigneaud ${ }^{6}$. It had a milk-ejecting potency equal to its oxytocic activity. The assay was based on two criteria, namely, the volume of milk ejected and the duration of the let-down action. These results point to oxytocin being the let-down hormone. However, they also raise the problem of why commercial preparations of pressor hormone with an oxytocic content stated by the makers to be less than five per cent should have a milk-ejecting activity of about five times their oxytocic activity.

The mode of action of the milk-ejecting hormone has in the past been assumed to be due to its acting on smooth-muscle fibres in the mammary gland. MacFarlan $\theta^{7}$ and Richardson ${ }^{8}$ have demonstrated the existence of myoepithelial cells so distributed in the mammary gland as to provide a mechanism by which the milk-containing alveoli can be squeezed and the contents expelled into the collecting ducts. These cells are also seen to run along the walls of the small ducts. As these ducts will be closed by the pressure of the surrounding filled alveoli prior to milking, their contraction or attempted contraction should result in an opening of the lumen on the duct. That such an opening may occur is suggested by the following evidence :

(1) When let-down occurs in the cow there is a sudden large rise in gland cistern pressure. This is followed by a small fall as the hormone is dissipated. Should milking be attempted, say, ten minutes after let-down, only 60 per cent or so of the normal milk yield will be obtained unless there is another release of hormone. The fall in pressure in the unmilked cistern is much smaller than it should be if the retained milk is held, due only to the alveoli relaxing and 'sucking back' the expelled liquid. These observations have been made by a number of workers as well as during work at this laboratory.

(2) In his studies on intramammary pressure at this laboratory, Mr. Phillips ${ }^{\ominus}$ has shown that during the period of action of the let-down hormone, the milk cistern of the cow is connected to a constantpressure source (such as contracting fibres around the elveolj). When the hormone goes out of action, the connexion to this constant-pressure source is broken.
(3) If, after let-down is evoked, sterile saline is run into the cistern of a cow to a pressure above that due to normal let-down, the injection of pituitary extract causes a fall in pressure. No change or a rise in pressure would be expected if the only action of the milk let-down hormone is the compression of the alveoli.

(4) In the case of the sow, which has no gland cistern, the flow of milk stops suddenly when the hormone is dissipated, almost as if a valve mechanism shuts when a certain threshold is reached.

In the light of this evidence, it is suggested that when the let-down hormone acts on the mammary gland it not only causes a contraction of the myoepithelium around the alveoli, so causing an increase in pressure within the latter, but also causes an opening of the small ducts due to the tightening of the longitudinal myoepithelial structures surrounding them. This causes a fall in duct impedance and a consequent rapid flow of the milk into the large ducts and the gland cistern.

W. G. WHITTLESTON

Ruakura Animal Research Station, Hamilton,

New Zealand. Sept. 11.

1 Schafer, A. E., J. Exp. Physiol., 8, 379 (1915).

8 Gaines, W. L., Amer. J. Physiol., 38, No. 2, 285 (1915)

${ }^{8}$ Ely, F., and Petersen, W. E., J. Dairy Sci., 24, No. 3, 211 (1941).

- Turner, C. W., cyclostyled notes on milk secretion, p. 89 (1938).

Turner, C. W., and Cooper, W. O., Endocrinol., 29, 320 (1941).

Livermore, A. H., and du Vigneaud, V., J. Biol. Chem., 180, 365 (1949).

- MeFarlane, D., Rennie, J. C., and Blackburn, P. S. (unpublished).

Richardson, K. C., Proc. Roy. Soc., B, 136, 30 (1949).

- Philips, D. S. M., Proc. N.Z. Animal Prod. Soc. (in the press).

\section{Milk Ejection following Electrical Stimulation of the Pituitary Stalk in Rabbits}

CONSIDERABLE indirect evidence exists ${ }^{1}$ for the involvement of the posterior pituitary gland in 'letdown' or ejection of milk. Experiments were performed to find the effect of electrical stimulation of the pituitary stalk on the mammary glands in six lactating rabbits. After overnight separation from their litters, the animals were anæsthetized with other, and a single teat-duct was cannulated and connected to a kymographic volume-recording system. A glass-insulated platinum wire electrode with $1 \mathrm{~mm}$. bare tip mounted on a stereotaxic instrument was used for stimulation. A standard stimulus of $1 \mathrm{~min}$. duration was adopted with 1.0 volt, 50 cycles sinewave alternating current.

Following stimulation, milk was seen to flow up the cannula after a latent period of about 25 sec., reaching a peak at 90 sec., and thereafter gradually subsiding to the original level over 4-7 min. The volume of milk ejected was $0 \cdot 5-1 \cdot 5$ c.c., representing approximately $1 / 48$ of the total ejection volume, since there are six ducts in each teat. Successive ejection responses were of diminishing magnitude, but as many as three responses could be elicited in a period of $30 \mathrm{~min}$. Intravenous injection of $200 \mathrm{mU}$. posterior pituitary extract gave similar ejection responses with a latent period of 10-15 sec.

These results offer strong support for posterior pituitary mediation of 'let-down'. They provide no clue to the mechanism initiating the response; but recent evidence ${ }^{2}$ suggests that the suckling stimulus activates the neurohypophysis in the normal un- 